\title{
Investigation Correlates of Chlamydia Anti-Body Testing and Hysterosalpingography among Women with Tubal Infertility
}

\author{
Afolabi Korede Koledade, Adebiyi Gbadebo Adesiyun \\ Obstetrics and Gynaecology Department, Ahmadu Bello University Teaching Hospital, Zaria, Nigeria \\ Email: akdade@yahoo.com
}

Received 8 October 2014; revised 8 November 2014; accepted 1 December 2014

Academic Editor: Yasam Kemal Akpak, Ankara Mevki Military Hospital, Turkey

Copyright (C) 2014 by authors and Scientific Research Publishing Inc.

This work is licensed under the Creative Commons Attribution International License (CC BY). http://creativecommons.org/licenses/by/4.0/

(c) ()

\section{Abstract}

Background: Chlamydia trachomatis infection is an important preventable cause of infertility. In women, up to $70 \%$ of genital infection with Chlamydia trachomatis are asymptomatic. In the management of infertility patients, a lot of clinicians or centres do not routinely screen for Chlamydia trachomatis infection. Hence all patients being investigated for infertility may potentially be at risk of tubal blockage in addition to non-tubal factor aetiology. Those with primary tubal blockage also are at risk of worsening of the blockage. Objective: To determine if there is a relationship between IgG and IgM Chlamydia antibody testing (CAT) and tubal factor infertility. Design: It was a cross sectional descriptive study. Method: The sera of 400 consecutive consenting infertile patients presenting to the gynaecological clinic of the Ahmadu Bello University Teaching Hospital, Zaria, Northern Nigeria were tested for Chlamydia antibodies using ELISA IgG and IgM kits produced by Diagnostic Automation, Inc., 23961 Craftsman Road, Suite D/E/F, Calabasas, CA 91302, USA. Results: Up to $264(66 \%)$ of the patients had tubal factor, $64(16 \%)$ had uterine, $56(14 \%)$ had ovarian, $50(12.5 \%)$ had male while $40(10 \%)$ had others. The causative factors were not mutually exclusive. The sero-prevalence of IgG and IgM Chlamydia trachomatis amongst patients with tubal infertility were $35.6 \%$ and $35.6 \%$ respectively. There was a stronger significant relation $(P=$ 0.008 ) between IgM sero-positivity which suggests recent infection and tubal infertility. The relation between IgG sero-positivity which suggest chronic infection and tubal infertility was also significant $(\mathrm{P}=\mathbf{0 . 0 3 6}$ ) but relatively lower. Conclusion: The sero-prevalence of Chlamydia trachomatis infection, IgG and IgM was significantly higher among the sub-population with tubal infertility. Chlamydia antibody testing (CAT) has predictive value for tubal infertility. 


\section{Keywords}

\section{Sero-Prevalence, Chlamydia trachomatis, IgG Antibodies, IgM Antibodies, Tubal Infertility}

\section{Introduction}

Tubal disease following PID is the commonest cause of infertility in Africa and this is caused by sexually transmitted infections, with Neisseria gonorrheae and Chlamydia trachomatis being the most common pathogens [1]. However, cervical infection with Chlamydia trachomatis has become an increasingly recognized problem in obstetrics and gynaecology. In women, up to $70 \%$ of genital infection with Chlamydia trachomatis are asymptomatic and among those with symptomatic pelvic inflammatory disease (PID) due to Chlamydia trachomatis, $20 \%$ may become infertile while $10 \%$ may have ectopic pregnancy [2]. Some reports have suggested that untreated maternal cervical chlamydial infection increases the risk of preterm delivery, PROM, stillbirth and postpartum endometritis and that $60 \%$ of neonates delivered vaginally to infected mothers develop conjunctivitis which, if untreated results in blindness [2].

Genital Chlamydia trachomatis is the commonest sexually transmitted infection (STI) today in Nigeria although majority of the infections are asymptomatic, sequelae such as PID, ectopic pregnancy and infertility are common [3] [4]. In high-risk settings, $2 \%$ to $5 \%$ of untreated women developed PID within approximately 2week period of testing positive for Chlamydia trachomatis [5]. Studies have also shown that sexually transmitted pathogens including non-ulcerative agents such as Chlamydia trachomatis may serve as biological co-factor for HIV infection [6] [7]. Chlamydia infections like other STI in general are primarily a woman's health care issue since the manifestations and consequences are more damaging to the reproductive health in women than in men; however, in men it causes Balanitis, Epididymitis, Prostatitis and Urethritis. Research on the link between Chlamydia trachomatis and male aspects of infertility has been much more limited. Timely management of sexual partners is essential for decreasing the risk for re-infection.

At the Ahmadu Bello University Teaching Hospital, Zaria in Nigeria, the protocol for the management of infertility does not include routine Chlamydia Antibody Testing or prophylactic antibiotics for Chlamydia trachomatis before hysterosalpingography evaluation.

This study sought to determine the aetiologic pattern of infertility amongst women that are positive for recent (IgM) and chronic (IgG) Chlamydia trachomatis infection with the view of finding if there is a positive correlation between Chlamydia trachomatis and hysterosalpingographic tubal abnormality. It also seeks to determine some reproductive health indices of the patients.

\section{Materials and Method}

The basic evaluation done for all infertility patients includes detailed history, physical examination, pelvic ultrasonography, hysterosalpingography, serum prolactin and semen analysis.

The study design is a descriptive cross-sectional laboratory based study of 400 consecutive consenting infertility patients.

Consecutive old and new patients presenting with infertility at the gynaecological clinic of ABUTH Zaria, Nigeria were selected and enrolled into the study after processing an informed written consent. Patients who had received either systemic or vaginal antibiotic therapy in the preceding six weeks were excluded from the study.

All eligible patients were serially numbered until the desired sample size was attained. Upon consenting to participate in the study, patients were given numbered proforma which they took to the side laboratory within the clinic where venopuncture was done. They were then interviewed using a standardized proforma. Patients whose aetiology of infertility was unknown as at the time of recruitment were followed up at the clinic to determine the likely cause(s) of infertility based on the outcome of their clinical evaluation. Referral letters and case notes were checked for additional information. This also helped to remove recall bias.

About $4 \mathrm{ml}$ of venous blood was collected from the patients into plain sample bottles at the side laboratory. The serum was extracted and samples taken to the main medical microbiology laboratory within 1 hour of collection where the samples were pooled in the refrigerator at a temperature of $2^{\circ} \mathrm{C}-8^{\circ} \mathrm{C}$ for not more than seven days. The sera were tested for Chlamydia antibodies using ELISA IgG and IgM kits produced by Diagnostic 
Automation, Inc., 23961 Craftsman Road, Suite D/E/F, Calabasas, CA 91302, USA. This has sensitivity of 96.1\% and specificity of $98.5 \%$. The IgG kit contained Microwell strips, Sample diluents, Calibrator, Negative control, Positive control, Washing concentrate, Enzyme conjugate, Tetramethylbenzidine (TMB) chromogenic solution substrate and Stop solution. The IgM kit had similar contents. The manufacturer's instructions were followed.

The test run was considered valid provided the following criteria are met:

The O.D value of the reagent blank against air from a microwell reader should be less than 0.250 .

If the O.D value of the calibrator is lower than 0.250 , the test is not valid and must be repeated.

The IgG index for negative and positive control should be in the range stated on the labels.

Interpretation of results:

Negative: IgG index of 0.90 or less are sero-negative for IgG antibody.

Equivocal: IgG index of 0.91 - 0.99 are equivocal. Samples should be retested.

Positive: IgG index of 1.00 or greater.

Result, validation and interpretation are similar using the IgM reagent kit.

Data analysis was carried out using Statistical Package for Social Science software version 20 and appropriate tests of association were conducted. A p-value of less than 0.05 was considered statistically significant. Ninetyfive percent confidence interval was used.

\section{Results}

The mean age of the patients was $31.1 \pm 5.7$ years. Up to 234 (58.5\%) of the patients were unemployed but only $6(1.5 \%)$ of their spouses were not gainfully employed. Most of the women 346 (86.5\%) had some form of western education, 176 (44\%) had tertiary education. Three quarters of the patients 294 (75\%) were married in a monogamous family type. Majority 368 (92\%) were in their first order of marriage.

The mean duration of infertility was $6.7 \pm 5.4$ years. The mean duration attendance of patients at the infertility clinic was $3.2 \pm 4.5$ years. Also, 274 (68.5\%) have previously consulted other government hospitals, private hospitals, prayer houses and or traditional healing houses on account of infertility.

Secondary infertility constituted the feature of majority of the patients, 246 (61.5\%).

There was history of symptoms of urogenital tract infection in 296 (74\%) of the patients out of which only 198 (66.9\%) got treated. The symptoms occurred singly or in combination (Figure 1).

Only $74(18.5 \%)$ had ever used any form of contraception in the past. Combined Oral Contraceptive Pills (COCP) 38 (51.4\%) were the commonest used, followed by Injectables 16 (21.6\%).

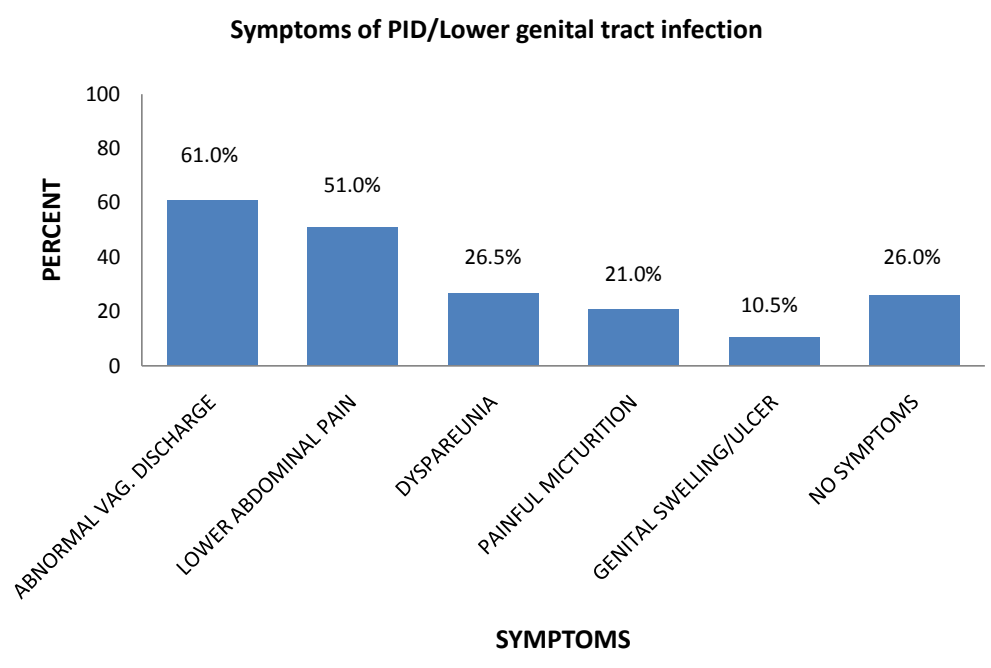

Figure 1. Distribution of symptoms among patients who had clinical features of urogenital infection. VAG. = Vaginal; PID = Pelvic Inflammatory Disease. Amongst the 296 (74\%) that had symptoms, abnormal vaginal discharge 224 (61\%) was the commonest followed by lower abdominal pain 204 (51\%). Dyspareunia was 106 (26.5\%) while painful micturition was 84 (21\%). Symptoms were not mutually exclusive. 
Up to 132 (33\%) of the 400 patients had had uterine evacuation before, mostly in private hospitals 102 (78.5\%). Manual vacuum aspiration (MVA) was the commonest method used. Out of the 400 patients, 100 (25\%) had MVA while $36(9 \%)$ had dilatation and curettage (D and C) and $2(0.5 \%)$ used misoprostol.

There was a statistically significant association $(\mathrm{P}=0.036)$ between sero-positivity for IgG Chlamydia trachomatis antibody and infertility due to tubal factor. Up to 94 (35.6\%) of the women that had tubal infertility were sero-positive for IgG Chlamydia trachomatis antibody. However, there was also a statistically significant association ( $(\mathrm{P}=0.008)$ between sero-positivity for IgM Chlamydia trachomatis antibody and infertility due to tubal factors 94 (35.6\%) (Figure 2 and Table 1).

\section{Discussion}

The sero-prevalence of IgG and IgM Chlamydia trachomatis amongst patients with tubal infertility were 35.6\% and $35.6 \%$ respectively. There appear to be a stronger association $(P=0.008)$ between IgM sero-positivity which suggests recent infection and tubal infertility. The association between IgG sero-positivity which suggest chronic infection and tubal infertility was also significant $(\mathrm{P}=0.036)$ but seem relatively weaker. The IgG Chlamydia trachomatis sero-prevalence amongst those with tubal infertility was similar to the prevalence of 38.3\% found by Tukur and Shittu et al. in a similar study in Zaria in 2002 [6] but was much lower than the IgG seroprevalence of $64.2 \%$ found amongst patients with tubal infertility in Benin [8]. This may be due to variation in the sexual and behavioural factors linked with infection with Chlamydia trachomatis. For example, the mean age for sexual debut in this study was $19.7 \pm 4.6$ years compared to an earlier sexual exposure of $17 \pm 3.6$ years in the Benin study. Also in the Benin study $84 \%$ of the patients had three or more life time sexual partners although this variable was not considered in this study.

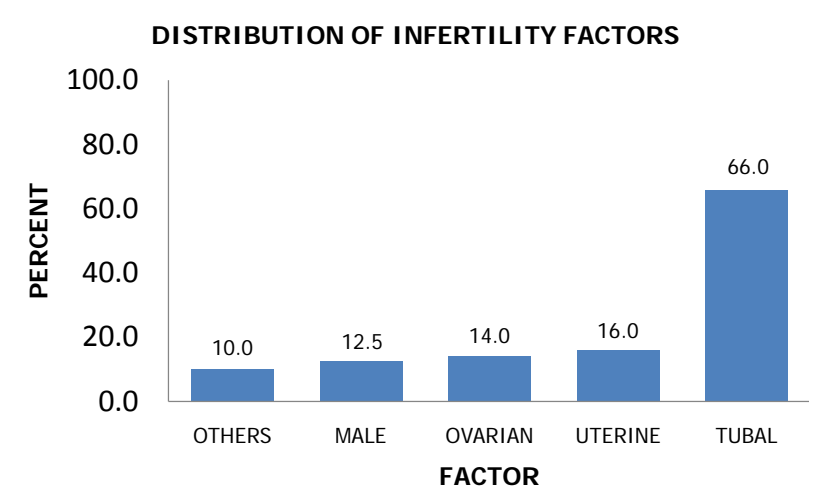

Figure 2. Distribution of causative factors for infertility among patients studied.

Table 1. Causative factors of infertility amongst patients that are seropositive for IgG and IgM.

\begin{tabular}{|c|c|c|c|c|}
\hline \multirow{2}{*}{ Causes of Infertility } & \multicolumn{2}{|c|}{ IgG Positive } & \multicolumn{2}{|c|}{ IgM Postive } \\
\hline & Frequency (\%) & Test statistics & Frequency (\%) & Test statistics \\
\hline $\begin{array}{c}\text { Tubal } \\
(\mathrm{N}=264)\end{array}$ & $94(35.6)$ & $\begin{array}{l}\chi^{2}=3.851 \\
\mathrm{P}=0.036\end{array}$ & $94(35.6)$ & $\begin{aligned} \chi^{2} & =0.6960 \\
P & =0.008\end{aligned}$ \\
\hline $\begin{array}{l}\text { Ovarian } \\
(\mathrm{N}=56)\end{array}$ & $14(25.0)$ & $\begin{array}{l}\chi^{2}=0.548 \\
P=0.309\end{array}$ & $12(21.4)$ & $\begin{array}{l}\chi^{2}=1.020 \\
P=0.221\end{array}$ \\
\hline $\begin{array}{l}\text { Uterine } \\
(\mathrm{N}=64)\end{array}$ & 14 (21.9) & $\begin{array}{l}\chi^{2}=1.483 \\
P=0.158\end{array}$ & $18(28.1)$ & $\begin{array}{l}\chi^{2}=0.035 \\
\mathrm{P}=0.520\end{array}$ \\
\hline $\begin{array}{c}\text { Male } \\
(\mathrm{N}=50)\end{array}$ & $10(20.0)$ & $\begin{array}{l}\chi^{2}=1.616 \\
P=0.151\end{array}$ & $10(20.0)$ & $\begin{array}{l}\chi^{2}=1.240 \\
P=0.193\end{array}$ \\
\hline $\begin{array}{c}\text { Others } \\
(\mathrm{N}=40)\end{array}$ & $8(20.0)$ & $\begin{array}{l}\chi^{2}=1.257 \\
\mathrm{P}=0.197\end{array}$ & $10(25.0)$ & $\begin{array}{l}\chi^{2}=0.216 \\
P=0.4341\end{array}$ \\
\hline
\end{tabular}

Degree of freedom $(\mathrm{df})=1$ for all; Note that the causes of infertility were not mutually exclusive. There was statistically significant association between tubal factor and IgG sero-positivity $(\mathrm{P}=0.036)$, and still between tubal factor and IgM sero-positivity $(\mathrm{P}=0.008)$. 
In addition to IgM sero-positivity in patients with tubal infertility, there was also IgM sero-positivity in 12 (21.4\%) of the patients with ovarian factor aetiology, 18 (28.1\%) of uterine factor, $10(20 \%)$ of male factor and 10 (25\%) of other aetiologies like unexplained infertility and intersex. This may suggest that patients with tubal infertility could have acquired this as a result of possible multiple re-infection with Chlamydia trachomatis and this may be associated with the severity of their tubal disease. Also patients with other causes of infertility could have been at risk of infection with Chlamydia trachomatis infection which might have led to tubal factor in addition to the original cause of infertility.

It is also noteworthy that the mean duration of infertility was $6.7 \pm 5.4$ years while the mean duration of attendance at our infertility clinic was $3.2 \pm 4.5$ years. This is however not surprising as tubal factor constituted the majority (66\%) of the patients. These re-emphasize the place of assisted reproductive technology (ART) in treatment of infertility especially tubal infertility but its accessibility and affordability has remained a challenge in sub-saharan Africa [9].

\section{Conclusions}

Tubal infertility constituted the majority (35.5\%) amongst patients who were sero-positive for Chlamydia trachomatis IgG and was statistically significant $(\mathrm{P}=0.036)$. Other aetiological factors found were ovarian $(25 \%)$, uterine (21.9\%), male (20\%) and others (20\%)

There was also a statistically significant association between tubal factor infertility and sero-positivity for $\operatorname{IgM}(\mathrm{P}=0.008)$.

\section{Recommendation}

Patients being investigated for infertility should be screened and treated for Chlamydia trachomatis infection. However in low resource areas where screening facilities are not available, patients there would benefit from empirical treatment for Chlamydia trachomatis before tubal patency assessment.

\section{References}

[1] Posada, C., Jonasson, J., et al. (1992) Prevalence of Urogenital Chlamydia Trachomatis Infection in EL-SalvadorInfection during Pregnancy and Perinatal Transmission. International Journal of STD \& AIDS, 3, 33-37.

[2] Moussavi, Z. and Behrouzi, R. (1996) The Prevalence of Chlamydia Infection among Pregnant Women Referred to Perinatal Clinics of Tehran University of Medical Sciences in IRAN. 25th Congress of Medical Women's International Association. http://www.regional.org.au/au/mwia/papers/full/20_moussavi.htm

[3] Centre for Disease Control and Prevention (2006) Guidelines for Treatment of Sexually Transmitted Diseases. Morbid/Mortal Weekly Report, 55, 11. http://www.cdc.gov/std/treatment/2006/rr5511.pdf

[4] Isibor, J.O., Ugbomoiko, D., et al. (2005) Detection of Chlamydia Antigen in Cervical Specimens from Antenatal Clinic Attendees in Benin City, Nigeria. African Journal of Clinical and Experimental Microbiology, 6, 208-211.

[5] Haggerty, C.L., Gottlieb, S.L., Taylor, B.D., Low, N., Xu, F. and Ness, R.B. (2010) Risk of Sequelae after Chlamydia Trachomatis Genital Infection in Women. The Journal of Infectious Diseases, 201, S134-S155.

[6] Tukur, J., Shittu, S.O. and Abdul, A.M. (2006) A Case Control Study of Active Genital Chlamydia trachomatis Infection among Patients with Tubal Infertility in Northern Nigeria. Tropical Doctor, 36, 14-16. http://www.ncbi.nlm.nih.gov/pubmed/16483420

[7] Rastogi, S., Kapur, S., et al. (1999) Chlamydia Trachomatis Infection in Pregnancy Risk Factor for an Adverse Outcome. British Journal of Biomedical Science, 56, 94-98.

[8] Omo-Aghoja, L.O., Okonofua, F.E., Larsen, U. and Bergstrom, S. (2007) Association of Chlamydia trachomatis Serology with Infertility in Nigerian Women. Journal of Obstetrics and Gynaecology Research, 33, 688-695.

[9] Cooke, I.D. (2007) The Globalization of Reproductive Technology. In: Kruger, T.F., van der Spuy, Z. and Kemper, B.D., Eds., Advances in Fertility Studies and Reproductive E Medicine, Cape Town Juticalpa, 234-240. 
Scientific Research Publishing (SCIRP) is one of the largest Open Access journal publishers. It is currently publishing more than 200 open access, online, peer-reviewed journals covering a wide range of academic disciplines. SCIRP serves the worldwide academic communities and contributes to the progress and application of science with its publication.

Other selected journals from SCIRP are listed as below. Submit your manuscript to us via either submit@scirp.org or Online Submission Portal.
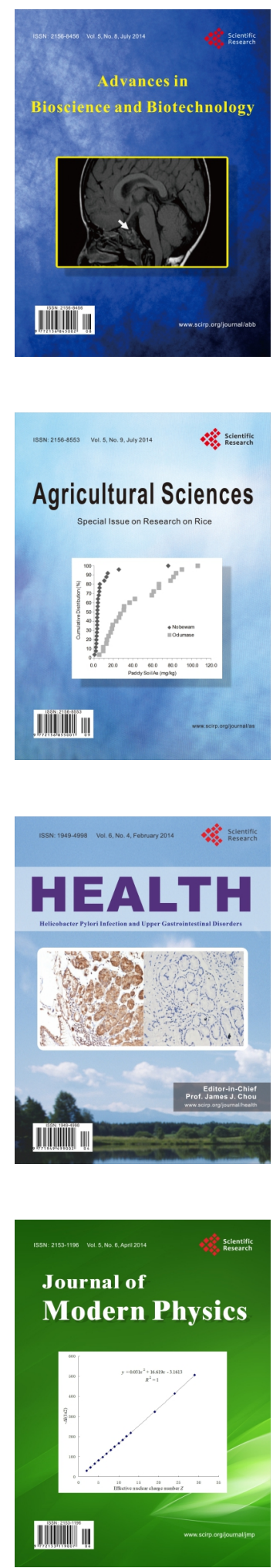
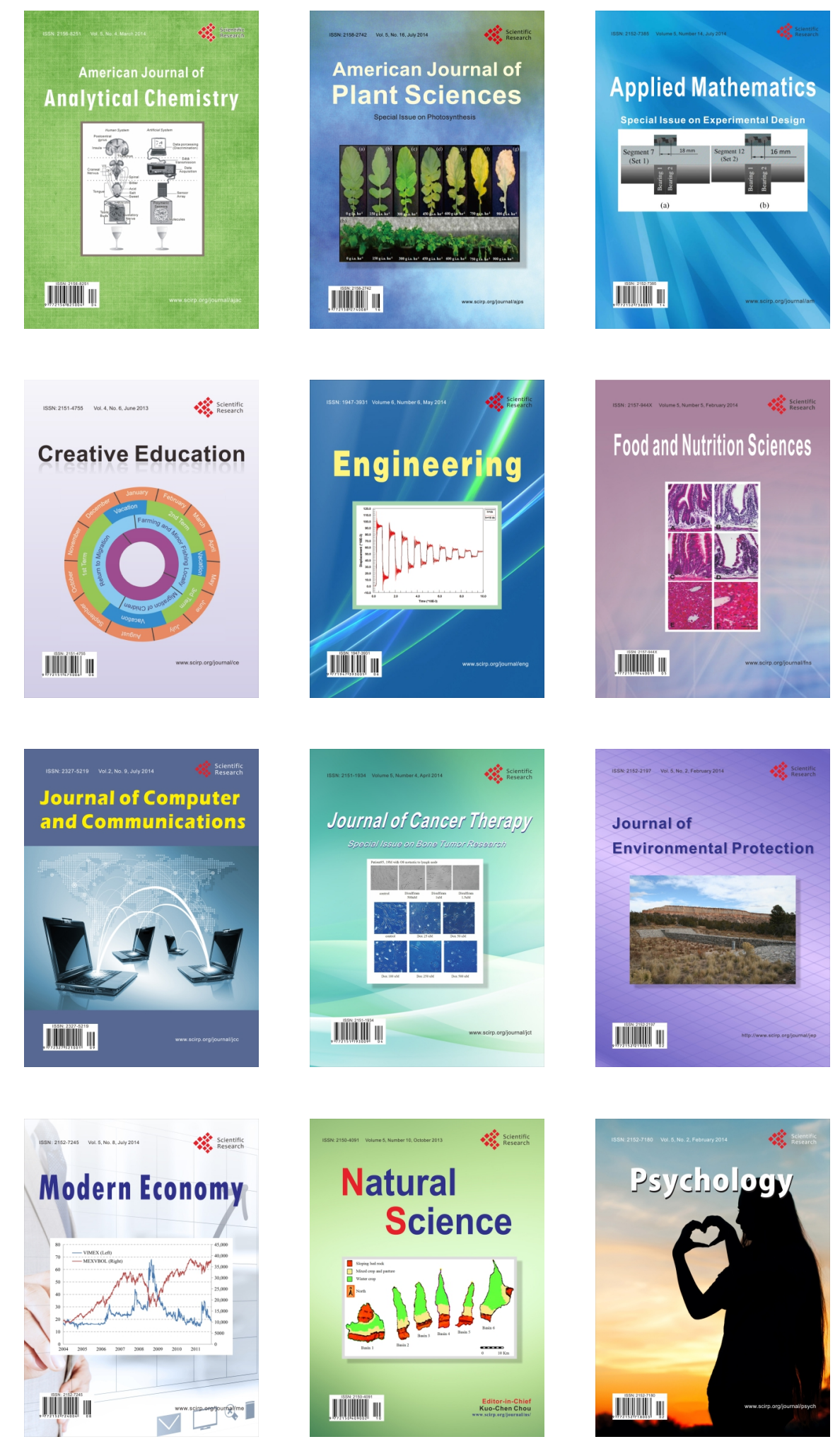